\title{
Association of the IL-6 -174G/C gene polymorphism with knee osteoarthritis in a Thai population
}

S. Honsawek ${ }^{1}$, B. Deepaisarnsakul ${ }^{2}$, A. Tanavalee ${ }^{3}$, P. Yuktanandana ${ }^{3}$, P. Bumrungpanichthaworn ${ }^{1}$, S. Malila ${ }^{1}$ and N. Saetan ${ }^{1}$

${ }^{1}$ Department of Biochemistry, Faculty of Medicine, Chulalongkorn University, Bangkok, Thailand

${ }^{2}$ Department of Clinical Laboratory, Taksin Hospital, Bangkok, Thailand

${ }^{3}$ Department of Orthopaedics, Faculty of Medicine,

Chulalongkorn University, Bangkok, Thailand

Presented at the Combined SICOT/RCOST 2009 Annual Meeting, Pattaya, Thailand, October 29 to November 1, 2009.

Corresponding author: S. Honsawek

E-mail: Sittisak.H@chula.ac.th

Genet. Mol. Res. 10 (3): 1674-1680 (2011)

Received January 11, 2011

Accepted July 27, 2011

Published August 8, 2011

DOI http://dx.doi.org/10.4238/vol10-3gmr1161

\begin{abstract}
Osteoarthritis is a chronic progressive degenerative joint disease characterized by age-related regressive change in articular cartilage. A single nucleotide polymorphism has been described at position -174 of the interleukin-6 (IL-6) promoter region, leading to three possible genotypes, GG, GC, and CC. We investigated a possible association of the IL-6 -174G/C gene polymorphism with knee osteoarthritis in a Thai population. Genotype distributions and allelic frequencies of the IL-6 -174G/C polymorphism were investigated in 115 knee osteoarthritis patients and 100 healthy controls. Genotyping was performed using PCR-RFLP. The genotype distribution of IL-6 was 79 GG, 36 GC, 0 CC in knee osteoarthritis patients and $88 \mathrm{GG}, 12 \mathrm{GC}, 0 \mathrm{CC}$ in controls. The frequency of the GC genotype in subjects with knee osteoarthritis was higher than in controls $(\mathrm{P}<0.001)$. Logistic regression analysis showed
\end{abstract}


that the GC genotype was independently associated with increased risk of knee osteoarthritis (odds ratio $=3.3,95 \%$ confidence interval $=1.6-6.9, \mathrm{P}=0.001)$. These findings suggest that the $-174 \mathrm{G} / \mathrm{C}$ polymorphism of the IL- 6 gene promoter plays a role in the pathogenesis of knee osteoarthritis.

Key words: Interleukin-6; Case-control study; Thai population; Single nucleotide polymorphism; Knee osteoarthritis

\section{INTRODUCTION}

Osteoarthritis $(\mathrm{OA})$ is a common cause of degenerative joint disease characterized by age-related regressive change in articular cartilage. This degenerative arthritis leads to loss of joint function and can result in substantial morbidity and disability in the elderly (Krasnokutsky et al., 2008). Knee osteoarthritis is the leading cause of severe pain, functional limitation, and musculoskeletal disability. The genetic etiology of OA is not entirely known. Previously, OA was recognized as a noninflammatory arthropathy. However, recent studies have demonstrated that an inflammatory process plays a role in the pathogenesis of OA (Loeser, 2009; Banks, 2010). Pro-inflammatory cytokines are implicated as potential mediators in the disease (Steinmeyer, 2004). Recently, the involvement of genetic factors has been extensively documented as well.

Interleukin-6 (IL-6), a phosphorylated glycoprotein containing 185 amino acids, is a multifunctional pro-inflammatory cytokine that plays an essential role in the regulation of the immune response, hematopoiesis, inflammation, and several metabolic disorders (Ishihara and Hirano, 2002). The human IL-6 gene is mapped to chromosome 7p21-24 with an upstream promoter consisting of 303 bp (Bowcock et al., 1988). Polymorphic variants in the promoter region of the IL-6 gene may be responsible for variations in transcription that subsequently affect circulating levels of this cytokine (Fishman et al., 1998). The best characterized of these polymorphisms is a single nucleotide polymorphism at position -174, upstream of the transcription start site, involving substitution of cytosine (C) for guanine $(\mathrm{G})$. A common $-174 \mathrm{G} / \mathrm{C}$ IL-6 gene polymorphism has been examined in a wide variety of diseases, including degenerative arthritis (Pola et al., 2005).

In this case-control study, we hypothesized that the $-174 \mathrm{G} / \mathrm{C}$ polymorphism of the IL-6 gene promoter would contribute to the susceptibility or severity of knee osteoarthritis. The purpose of this study was to investigate the association between the IL- $6-174 \mathrm{G} / \mathrm{C}$ polymorphism and radiographic signs of osteoarthritis of the knee in Thai patients.

\section{MATERIAL AND METHODS}

\section{Subjects}

This case-control study was approved by the Institutional Review Board on Human Research of the Faculty of Medicine, Chulalongkorn University. The present study was conducted in compliance with the guidelines of the Declaration of Helsinki. All subjects gave written informed consent prior to their participation in the study.

A total of 115 patients aged 55 to 78 years diagnosed with primary knee osteoarthritis 
(97 females and 18 males; mean age $65.5 \pm 1.1$ years) and 100 control individuals ( 85 females and 15 males; mean age $63.8 \pm 2.1$ years) were recruited in this study. Participants were excluded on the basis of having arthropathy due to gout, pseudogout, rheumatoid arthritis, systemic lupus erythematosus, psoriasis, hemochromatosis, previous knee injury, or previous joint infection. Patients with any systemic inflammatory or autoimmune disorders, or any type of malignant or chronic illness were not included in this study. The severity of the disease was determined using weight-bearing anteroposterior radiographs of the affected knee. Knee radiographs were evaluated according to the Kellgren and Lawrence (1957) classification: grade 1 , doubtful narrowing of joint space and possible osteophytic lipping; grade 2, definite osteophytes and possible narrowing of joint space; grade 3, moderate multiple osteophytes, definite narrowing of joint space, some sclerosis and possible deformity of bone contour; grade 4, large osteophytes, marked narrowing of joint space, severe sclerosis and definite deformity of bone contour. The grading scale used for analysis was the higher of the two knees.

\section{Laboratory methods}

Peripheral venous blood samples of $3 \mathrm{~mL}$ were drawn from each individual by standard venipuncture. Genomic DNA was isolated from buffy coats by using the commercially available Qiagen Blood Cell and Culture Midi kit. Polymerase chain reaction (PCR) amplifications were performed for the $-174 \mathrm{G} / \mathrm{C}$ single nucleotide polymorphism (SNP) using the published primer set: 5'-TGACTTCAGCTTTACTCTTTGT-3' (sense primer) and 5'-CTGATTGGAAACCTTATTAGG-3' (antisense primer). The conditions of the PCR were as previously described (Fernández-Real et al., 2000). The amplified sequence was digested by $S f a$ NI restriction enzyme (New England BioLabs, Beverly, MA, USA) at $37^{\circ} \mathrm{C}$ overnight. The PCR products were separated on $2 \%$ agarose gel electrophoresis and visualized by ethidium bromide staining.

\section{Statistical analysis}

Statistical analysis was performed using the Statistical Package for Social Sciences (SPSS) software, version 16.0 for Windows. Demographic and clinical data were compared between groups by the chi-square test and $t$-test. Genotype distributions and allelic frequencies were compared by the chi-square test. Odds ratios (OR) were calculated with $95 \%$ confidence interval (CI). Allele and genotype proportions were evaluated for Hardy-Weinberg equilibrium. $\mathrm{P}<0.05$ was considered as indicating statistical significance.

\section{RESULTS}

Characteristics of the population studied and the number of individuals in each group are illustrated in Table 1. There were no significant differences between groups in terms of age, gender, and mean body mass index (BMI). In knee OA patients, the mean age was 65.5 \pm 1.1 years. In healthy controls, the mean age was $63.8 \pm 2.1$ years $(\mathrm{P}=0.4)$. The female/ male ratio was $97 / 18$ in patients with knee $\mathrm{OA}$ and $85 / 15$ in controls $(\mathrm{P}=0.5)$. Furthermore, average BMI value was not significantly different between groups, $25.7 \pm 3.3 \mathrm{~kg} / \mathrm{m}^{2}$ in knee OA patients and $25.2 \pm 2.5 \mathrm{~kg} / \mathrm{m}^{2}$ in controls, respectively $(\mathrm{P}=0.2)$. 


Table 1. Characteristics of the study population.
\begin{tabular}{lccc} 
\\
\hline \\
\hline Control group (N =100) & OA group (N=115) & P \\
\hline Age (years) & $63.8 \pm 2.1$ & $65.5 \pm 1.1$ & 0.4 \\
Female $/$ male & $85 / 15$ & $97 / 18$ & 0.5 \\
Body mass index $\left(\mathrm{kg} / \mathrm{m}^{2}\right)$ & $25.2 \pm 2.5$ & $25.7 \pm 3.3$ & 0.2 \\
\hline
\end{tabular}

$\mathrm{OA}=$ osteoarthritis.

An example of an electrophoretic gel showing PCR product digestion with $S f a \mathrm{NI}$ is given in Figure 1. The GG genotype corresponded to the presence of 140- and 58-bp fragments. The GC genotype corresponded to the presence of 198-, 140-, and 58-bp fragments. The CC genotype corresponded to a 198-bp fragment. Table 2 shows the IL- 6 gene polymorphism genotype frequency. GG was the most frequent genotype in cases and controls. In 115 patients with knee OA, the genotype distribution was 79 GG, 36 GC, 0 CC. Such distribution was significantly different from that observed in controls $(\mathrm{N}=100)$ : $88 \mathrm{GG}, 12 \mathrm{GC}, 0 \mathrm{CC}$. The frequency of the GC genotype in subjects with knee OA was 2.5-fold greater than in controls (31.3 vs $12 \%$; $\mathrm{P}<0.001)$. On the contrary, the frequency of the GG genotype was higher in controls than in knee OA patients but this difference was not statistically significant (88.0 vs $68.7 \% ; \mathrm{P}=0.4)$. The $\mathrm{G}$ allele was observed in $84.4 \%$ of knee OA patients and in $94 \%$ of controls, whereas the $\mathrm{C}$ allele was found in $15.6 \%$ of patients and in $6 \%$ of controls $(\mathrm{P}<0.001)$.

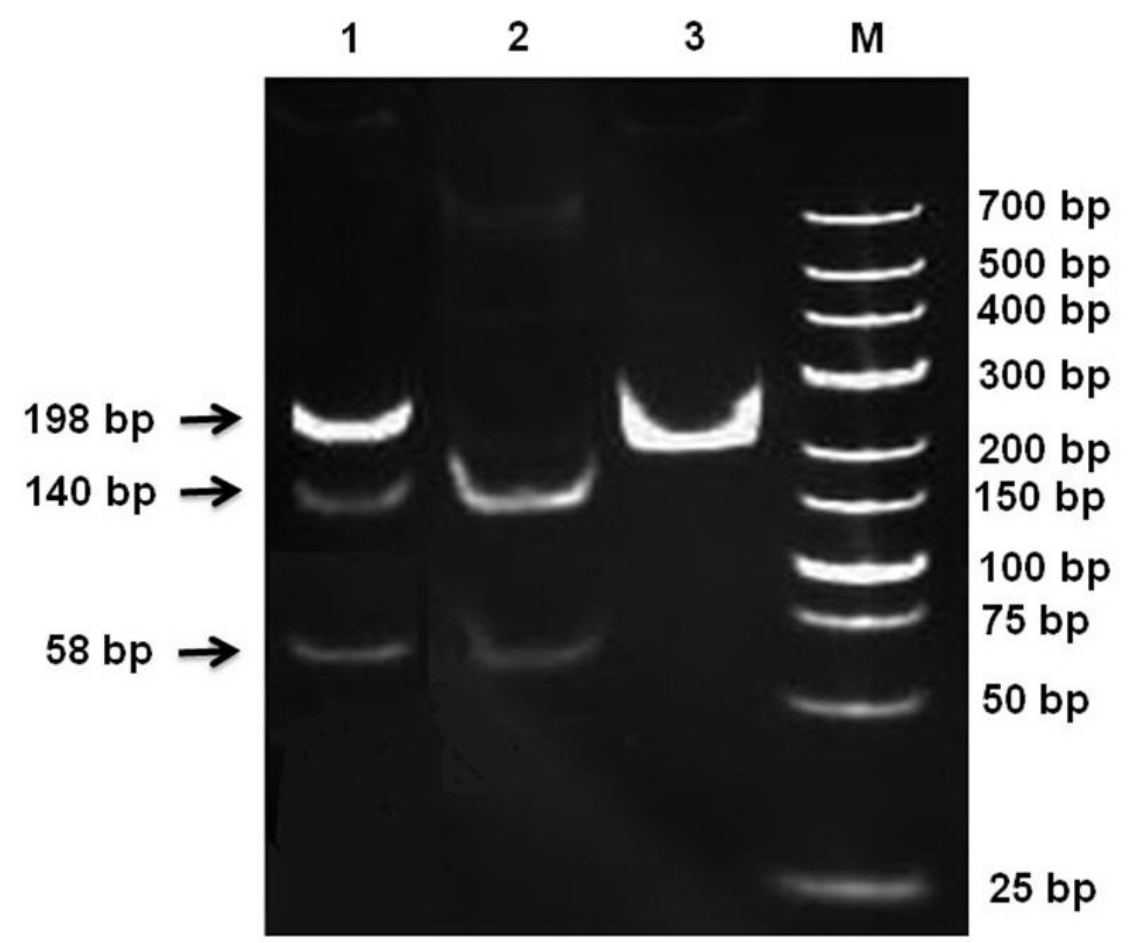

Figure 1. Genotypes of interleukin-6 polymorphism on 2\% agarose gel electrophoresis with ethidium bromide staining and ultraviolet light transillumination. Lane 1 = Heterozygous GC; lane 2 = homozygous GG; lane $3=$ homozygous $\mathrm{CC}$; lane $\mathrm{M}=$ molecular weight DNA ladder standard. 
Table 2. Genotype distribution and allelic frequency of the IL-6 -174G/C polymorphism in patients with knee osteoarthritis (OA) and controls.

\begin{tabular}{lccc}
\hline & Control group (N=100) & OA group (N=115) & P \\
\hline Genotype & & & 0.4 \\
G/G (N, \%) & $88(88.0)$ & $79(68.7)$ & $<0.001$ \\
G/C (N, \%) & $12(12.0)$ & 0 & - \\
C/C (N, \%) & 0 & $196(31.3)$ & - \\
Allele & $188(94.0)$ & $36(15.6)$ & 0.001 \\
G (N, \%) & $12(6.0)$ & & \\
C (N, \%) & &
\end{tabular}

A logistic regression analysis (Table 3 ) revealed that the GC genotype was independently associated with increased risk of the knee osteoarthritis $(\mathrm{OR}=3.3,95 \% \mathrm{CI}=1.6-6.9, \mathrm{P}=0.001)$.

Table 3. Risk factors for knee osteoarthritis based on logistic regression analysis.

\begin{tabular}{lcll}
\hline & Odds ratio & $95 \% \mathrm{CI}$ & $\mathrm{P}$ \\
\hline G/G genotype & 0.3 & $0.1-0.6$ & 0.5 \\
G/C genotype & 3.3 & $1.6-6.9$ & 0.001 \\
Gender, female & 1.9 & $1.0-3.8$ & 0.06 \\
\hline
\end{tabular}

$\mathrm{CI}=$ confidence interval.

\section{DISCUSSION}

Cytokines are essential regulators in the pathogenesis of synovitis and cartilage destruction associated with OA (Goldring and Goldring, 2004). Difference in levels of various cytokines among different individuals can be a plausible explanation for differences in disease susceptibility and severity. These variations are principally attributable to polymorphism in the genes encoding cytokines. A number of studies have suggested that OA is a polygenic disease regulated by the expression of genetic factors. In addition, cytokine genes have a potential role in controlling the catabolic/anabolic balance of articular cartilage. Several cytokines are involved in cartilage metabolism and are synthesized by synovial cells and cartilage chondrocytes, among them IL-6 (Mohtai et al., 1996).

IL-6 is believed to be one of the crucial factors in joint destruction, being a pleiotropic pro-inflammatory cytokine that is markedly upregulated at times of tissue inflammation. A significant elevation in the expression of IL-6 mRNA has been observed in OA-affected cartilage, and the IL-6 levels in serum and synovial fluid have been documented to be increased among OA patients (Kaneko et al., 2000). Moreover, human recombinant IL-6 has been demonstrated to enhance human recombinant IL-1 $\beta$-induced proteoglycan degradation and to inhibit chondrocyte proliferation (Jikko et al., 1998). Known variations within the IL-6 gene have been repeatedly screened in a number of association studies. Gene polymorphisms involved in the development of knee osteoarthritis have been identified. According to the reports, a common guanine/cytosine polymorphism in the promoter region of the IL- 6 gene at position -174, influencing the degree of IL-6 expression (Fishman et al., 1998), has been described in association with chronic inflammatory disorders and/or arthritis (Kämäräinen et al., 2008). Whether 
the IL-6 genetic polymorphism at the -174 region influences the susceptibility or severity in patients with knee osteoarthritis is not fully understood. To address this issue, we analyzed the influence of the IL-6 -174G/C polymorphism on the risk of knee OA in the Thai population.

In this case-control study, our findings demonstrated that the GC genotype of the $-174 \mathrm{G} / \mathrm{C}$ polymorphism of the IL-6 gene promoter determined a higher risk for knee osteoarthritis. However, this is not consistent with the results observed in a previous study with regard to the hip joint, indicating that the $\mathrm{CC}$ genotype determines a lower risk profile for osteoarthritic disease. This discrepancy may be attributed to intrinsic site-related differences between the hip and knee joints. In addition, it has been reported that gene associations with osteoarthritis are dependent on gender and ethnicity, and are possibly often site-specific (Valdes et al., 2007). The difference in ethnicity may be related.

The present study examines only the effects of a single SNP on the presence of OA. A limitation of this study is the lack of other polymorphisms of the IL-6 gene. Haplotype analysis is needed to elucidate the functional variation responsible for IL-6 expression and may provide further knowledge on the pathways responsible for the association of IL-6 gene with OA. Another weakness is the lack of data regarding the level and source of IL-6. More research is needed for future in vitro and in vivo studies of representative cells and tissues to gain insight into IL-6 production and expression.

It is conceivable that a disease that has been described mainly as noninflammatory shows a relationship with polymorphisms in genes that encode inflammatory cytokines. IL-6 is one of the important regulators in inflammatory reactions, and it is noteworthy that polymorphic cytokine genes enhance localized inflammatory reactions in the joints (Krenn et al., 1999), which in turn promote the deregulated cytokine expression in articular chondrocytes. However, the pathogenesis of $\mathrm{OA}$ is multifactorial, with a variety of environmental and genetic factors acting in concert, and further investigation will be required to identify the additional genes involved and the triggers initiating the disease.

In summary, we provide evidence that the $-174 \mathrm{G} / \mathrm{C}$ polymorphism of the IL-6 gene promoter is a risk factor for knee OA. Although additional studies are necessary, our results support the hypothesis that IL-6 is essential in the pathophysiology of OA.

\section{ACKNOWLEDGMENTS}

Research supported by the Thailand Research Fund, Commission on Higher Education and National Research Council of Thailand. The authors are grateful to Dr. Padet Siriyasathien and Dr. Kanok Preativatanyou and Chulalongkorn Medical Research Center (ChulaMRC) for kindly providing facilities. This study was also facilitated by the Ratchadapiseksompotch Fund, Faculty of Medicine, Chulalongkorn University.

\section{REFERENCES}

Banks SE (2010). Erosive osteoarthritis: a current review of a clinical challenge. Clin. Rheumatol. 29: 697-706. Bowcock AM, Kidd JR, Lathrop GM, Daneshvar L, et al. (1988). The human "interferon-beta 2/hepatocyte stimulating factor/interleukin-6" gene: DNA polymorphism studies and localization to chromosome 7p21. Genomics 3: 8-16.

Fernández-Real JM, Broch M, Vendrell J, Richart C, et al. (2000). Interleukin-6 gene polymorphism and lipid abnormalities in healthy subjects. J. Clin. Endocrinol. Metab. 85: 1334-1339.

Fishman D, Faulds G, Jeffery R, Mohamed-Ali V, et al. (1998). The effect of novel polymorphisms in the interleukin-6 (IL-6) gene on IL-6 transcription and plasma IL-6 levels, and an association with systemic-onset juvenile chronic 
arthritis. J. Clin. Invest. 102: 1369-1376.

Goldring SR and Goldring MB (2004). The role of cytokines in cartilage matrix degeneration in osteoarthritis. Clin. Orthop. Relat. Res. S27-S36.

Ishihara K and Hirano T (2002). IL-6 in autoimmune disease and chronic inflammatory proliferative disease. Cytokine Growth Factor Rev. 13: 357-368.

Jikko A, Wakisaka T, Iwamoto M, Hiranuma H, et al. (1998). Effects of interleukin-6 on proliferation and proteoglycan metabolism in articular chondrocyte cultures. Cell Biol. Int. 22: 615-621.

Kämäräinen OP, Solovieva S, Vehmas T, Luoma K, et al. (2008). Common interleukin-6 promoter variants associate with the more severe forms of distal interphalangeal osteoarthritis. Arthritis Res. Ther. 10: R21.

Kaneko S, Satoh T, Chiba J, Ju C, et al. (2000). Interleukin-6 and interleukin-8 levels in serum and synovial fluid of patients with osteoarthritis. Cytokines Cell Mol. Ther. 6: 71-79.

Kellgren JH and Lawrence JS (1957). Radiological assessment of osteo-arthrosis. Ann. Rheum. Dis. 16: 494-502.

Krasnokutsky S, Attur M, Palmer G, Samuels J, et al. (2008). Current concepts in the pathogenesis of osteoarthritis. Osteoarthritis Cartilage 16 (Suppl 3): S1-S3.

Krenn V, Hensel F, Kim HJ, Souto Carneiro MM, et al. (1999). Molecular $\operatorname{IgV}(\mathrm{H})$ analysis demonstrates highly somatic mutated B cells in synovialitis of osteoarthritis: a degenerative disease is associated with a specific, not locally generated immune response. Lab. Invest. 79: 1377-1384.

Loeser RF (2009). Aging and osteoarthritis: the role of chondrocyte senescence and aging changes in the cartilage matrix. Osteoarthritis Cartilage 17: 971-979.

Mohtai M, Gupta MK, Donlon B, Ellison B, et al. (1996). Expression of interleukin-6 in osteoarthritic chondrocytes and effects of fluid-induced shear on this expression in normal human chondrocytes in vitro. J. Orthop. Res. 14: 67-73.

Pola E, Papaleo P, Pola R, Gaetani E, et al. (2005). Interleukin-6 gene polymorphism and risk of osteoarthritis of the hip: a case-control study. Osteoarthritis Cartilage 13: 1025-1028.

Steinmeyer J (2004). Cytokines in osteoarthritis-current status on the pharmacological intervention. Front. Biosci. 9: 575-580.

Valdes AM, Loughlin J, Oene MV, Chapman K, et al. (2007). Sex and ethnic differences in the association of ASPN, CALM1, COL2A1, COMP, and FRZB with genetic susceptibility to osteoarthritis of the knee. Arthritis Rheum. 56: $137-146$. 